\title{
AVI TRANSPONDER
}

\author{
SHOICHI KAMATA, YOSHINORI KIMURA and JOHJI SAKURAGI
}

\author{
Toshiba Corporation, 1, Toshiba-cho, Komukai, Saiwai-Ku, Kawasaki 210, Japan
}

\begin{abstract}
A newly developed AVI transponder is to be used in Automati Vehicle Identification (AVI) system, which provides identification of moving vehicles passing by a certain location as to its registration number, and classification etc. This AVI system is characterized by the fact that it utilizes cerami resonators in the composition of a transponder identification code, and a single microwave carrier to convey an interrogation signal to, and a response signal from, that transponder.

The transponder is a completely passive device free of any power supply, and operates as a "Reflector". Overall external dimensions of transponder are $7 \times 10 \times 1 \mathrm{~cm}$ with a weight of 150 grams, the unit is compact, light weight and can be mounted easily on the vehicle, and is easy to carry.

All of the circuitry required for the transponder is provided in the form of microstrip lines utilizing MIC (Microwave Integrated Circuit) technology on the PTFE-glass fibre laminate substrate. The transponder circuitry, which consists of printed antenna, microwave modem circuit and medium frequency circuit, is patterned on both surfaces of the single substrate. In this paper, operation principle, circuitry and construction of the transponder will be described.
\end{abstract}

\section{INTRODUCTION}

In recent years, in step with the significant progress of highway and railroad transportation systems, high speed and high volume of traffic has become the general trend. This trend has brought about an urgent need for a means to automatically identify vehicles while in motion, as to their type or vehicle number.

Heretofore, the authors have developed ACI (Automatic Car Identification) system for railroads utilizing electromagnetic induction. ${ }^{1,2}$ This system has been proven as practical and its usefulness is being assessed. However, there is an inherent drawback to this system in that the transponder cannot be attached directly to a metal surface. Another drawback lies in the short distance of several decimeters, permissible between the road-side loop antenna and the transponder's loop. Such drawbacks have practically precluded application of electromagnetic systems, in the identification of automotive vehicles and containers in motion. Also in the past, several systems utilizing light rays have been developed ${ }^{3-5}$ and a few have been put into practical usage; however this system has the drawback of being affected by climatic and environmental conditions such as snow, and dust etc.

With the above described background, the advent of AVI (Automatic Vehicle Identification) systems utilizing microwaves with their inherent advantages, and the development of such systems have come to be conducted on a world-wide basis. ${ }^{6-8}$

These microwave systems can be grouped into those utilizing microwave cavity resonators, and those utilizing active elements. The former group usually utilizes a plurality of cavity resonators and thereby possesses, the disadvantage of becoming bulky, and of having difficulty in changing their encoding. On the other hand, the latter group has the problem of supplying power to the active elements of the transponder, either by transmitting large amounts of microwave power in order to supply the necessary DC power, or by equipping the transponders with batteries.

The newly developed microwave AVI system ${ }^{9}$ is characterized by the transponder. This new transponder has the advantages that it is a completely passive device, small in size, of flat construction, light in weight, and has a considerably long operation range from the interrogator antenna. This has enabled the transponder to provide an ideal system for the identification of automotive vehicles, rail cars and containers.

\section{GENERAL DESCRIPTION OF THE SYSTEM}

This system utilizes a combination of "ceramic resonators" to buildup the "encoded" identification 
code of a passive transponder, and the transmission of the interrogating and responding information is achieved through a microwave carrier. Use of ceramic resonators as identification elements has been put into practice in the past in systems using electromagnetic induction coupling. Figure 1(a) shows the basic configuration of such a system. In this system the transponder consists of a plurality of ceramic resonators with different resonance frequencies, and the identification code consists of a particular combination of the resonance frequencies. First the interrogator emits an interrogation signal consisting of a sweep-frequency through its transmitting loop-coil, which is then received by the transponder and impressed on its resonators. At this instant the resonators are excited and driven to resonate at their own resonance frequencies, and this generated response signal is next received by the loop-coil. The resonance frequencies in the received signal are detected and the encoded code is identified. On the other hand, in the microwave carrier system, the signal sent and received through the inductively coupled loop-coils in the electro-magnetic system is done so by means of a microwave carrier, so that a large increase in the distance between the interrogator antenna and transponder is made possible.

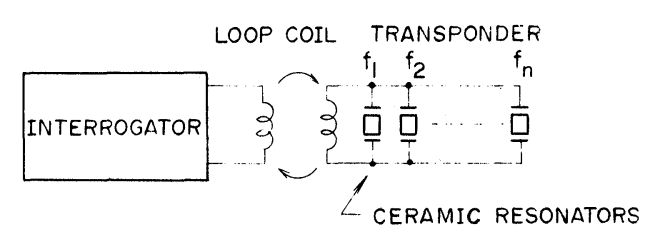

(a) ELECTRO-MAGNETIC COUPLING SYSTEM

ANTENNA ANTENNA TRANSPONDER

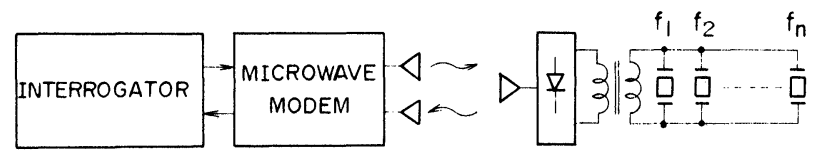

\section{(b) MICROWAVE AVI SYSTEM}

FIGURE 1 Comparison of system configuration.

The basic concept of this system is shown in Figure 1(b). Here, the loop-coil of the electro-magnetic system, is replaced by both the microwave modulator/demodulator and microwave antenna. This

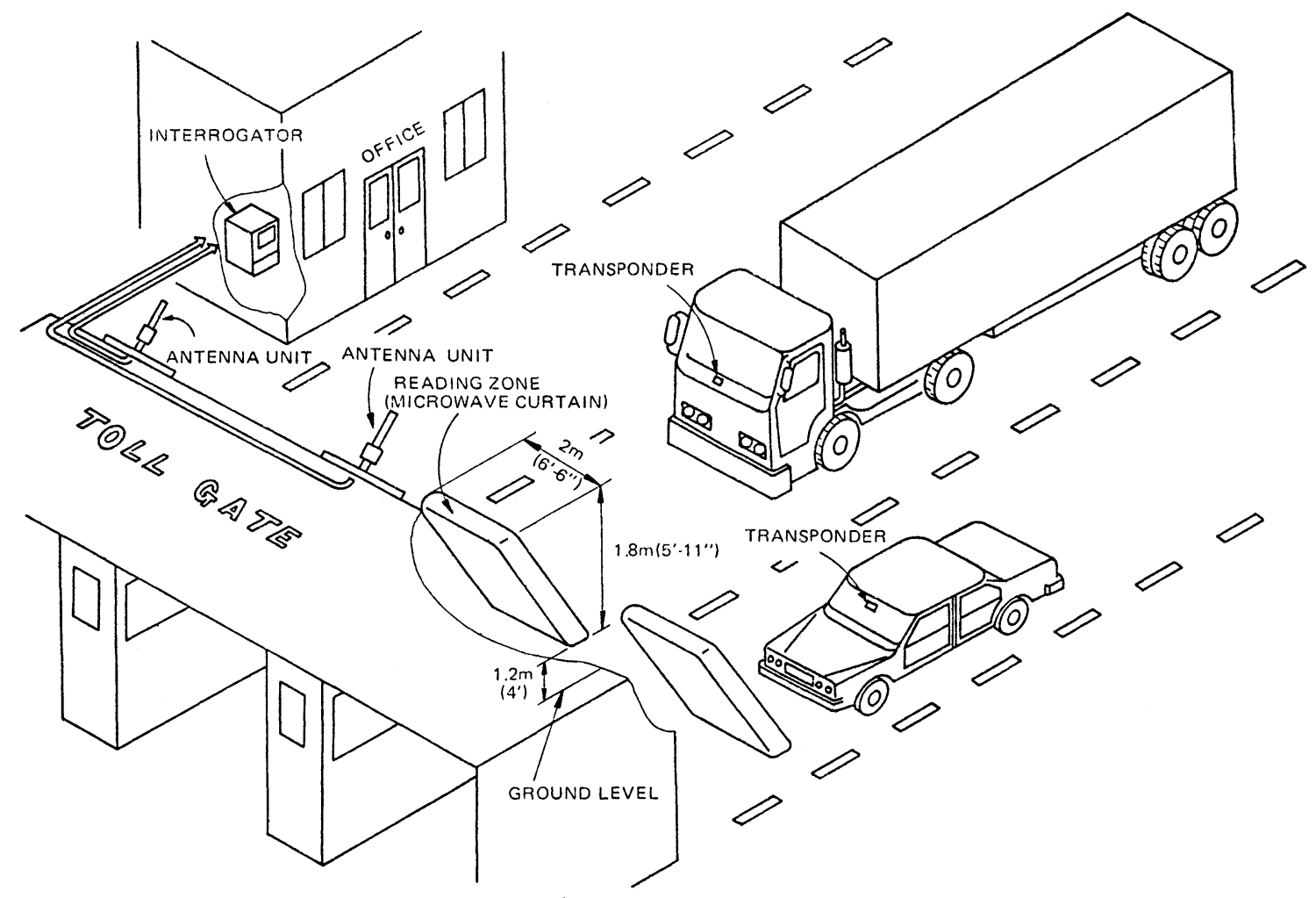

FIGURE 2 System overview. 
system possesses the following advantages:

1) Identification over a considerable distance and range is possible.

2) The transponder is of the completely passive type, is compact, will withstand severe vibrations and shocks and has high reliability under adverse environmental conditions.

3) The transponder can be attached directly to a metal surface.

4) Because of the microwave carrier, complete isolation from electrical engine-noise is possible.

5) The RF microwave power can be relatively small.

Figure 2 shows an example of this AVI system. The system is applied for non-stop toll collection system, in which the transponder is mounted inside the windshield of a vehicle, and the interrogator antenna is installed on the canopy of a toll gate.

\section{OPERATION OF THE SYSTEM}

Operation of this system is shown in the block diagram of Figure 3. The system consists of the interrogating system; made-up of the interrogator and the antenna unit, and the transponder. System operation is described according to signal flow.

\subsection{Transmission of the Interrogating Signal}

A sweep-frequency signal $f_{s}$, generated by a sweep-frequency signal generator, modulated the microwave carrier $f_{0}$ by the modulator, and produces the microwave interrogation signal containing frequency components such as $f_{0}$ and $f_{0} \pm f_{s}$. This microwave interrogation signal is amplified to a suitable level and transmitted through the transmitting antenna toward the transponder.

\subsection{Response of the Transponder}

The transmitted signal is received through the antenna of the transponder and is fed to the diode. As a result of nonlinearity of the diode, the sweep-frequency signal $f_{s}$ is demodulated and is applied to the resonators having different resonance frequencies. At this instant, each resonator starts to resonate at its own resonance frequency and produces the "ringing" signal having its own resonance frequency $f_{n}(n=1,2, \ldots, n)$. These ringing signals are again applied to the diode being excited by the microwave carrier $f_{0}$, to cause components, $f_{0} \pm f_{1}, f_{0} \pm f_{2}, \ldots, f_{0} \pm f_{n}$ to be generated. This forms the response signal which is transmitted through the transponder's antenna toward the receiving antenna of the interrogator.

\subsection{Receiving the Response Signal}

The response signal reflected by the transponder is received by the receiving antenna of the interrogator and is fed to the demodulator. The signal is demodulated at the demodulator which performs "homodyne detection" using the carrier frequency $f_{0}$ as the reference, and ringing signals with components $f_{1}$, $f_{2}, \ldots, f_{n}$ is obtained.

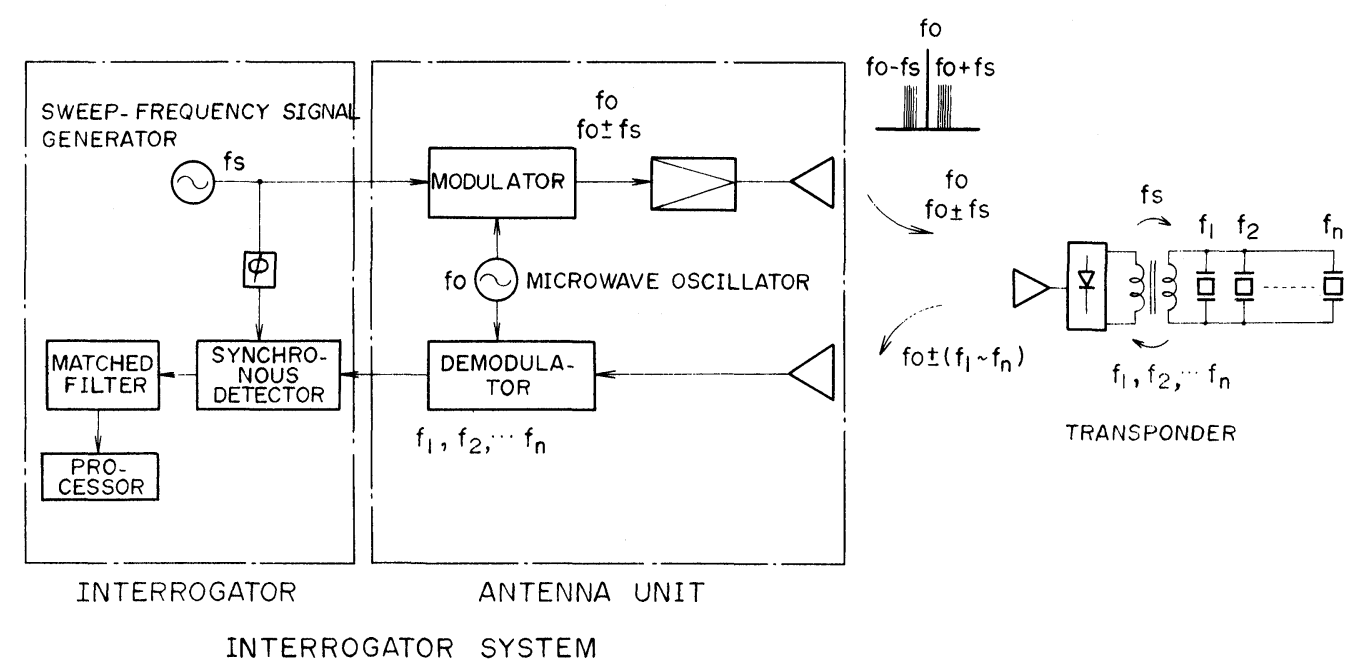

FIGURE 3 Microwave AVI system block diagram. 


\subsection{Detection of Resonance Frequencies of the Transponder}

In order to effectively detect the resonance points of the transponder, those ringing signals are processed by using a synchronous detector, Figure 4 shows the wave forms during this detection process. The ringing signals which have frequencies $f_{1}, f_{2}, \ldots, f_{n}$ are synchronously detected by using sweep-frequency signal $f_{s}$ as the reference, and are converted to "ringing beat" signal having the frequencies $f_{s}-f_{1}, f_{s}-f_{2}$, $\ldots, f_{s}-f_{n}$, respectively. The " $k$ " th resonating point's ringing beat signal's waveform can be represented by the following equation.

$$
\mathrm{e}_{b}=\mathrm{V} \exp \left[\omega_{k}\left(t-\tau_{k}\right) / 2 Q_{L}\right] \cdot \exp \left[-j \pi h\left(t-\tau_{k}\right)^{2}\right.
$$

where,

$V$ : constant

$\omega_{k}$ : “ $k$ "th resonance angular frequency

$\tau_{k}$ : " $k$ "th resonance starting time

$Q_{L}: Q$ of the transponder's resonance circuit

$h$ : sweep speed of the sweep-frequency

The waveform takes on the same shape for all of the resonance points, thus, these waveforms are converted, one by one into information pulses by means of a "matched filter". These information pulses are located in a row corresponding to the resonance points on the sweep time axis. By processing these pulses, identification can be achieved, of each particular code derived from the combinations of resonance frequencies of the transponder.

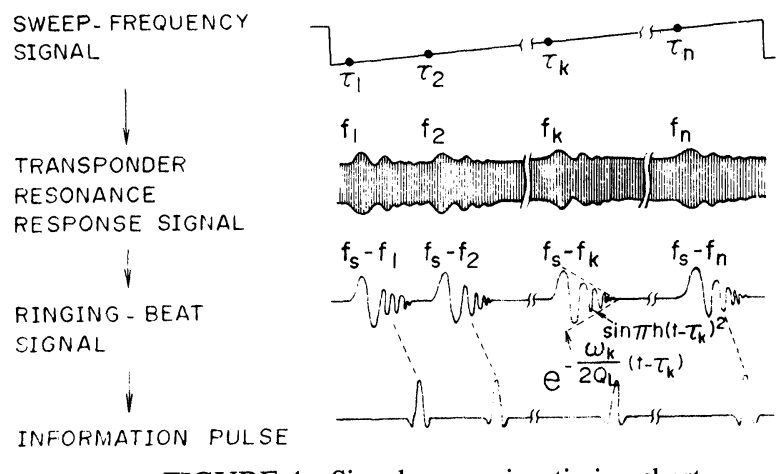

FIGURE 4 Signal procession timing chart.

\section{TRANSPONDER}

The transponder used in this system is a completely passive device free of any power supply. It receives the interrogating signal and reflects it after giving particular

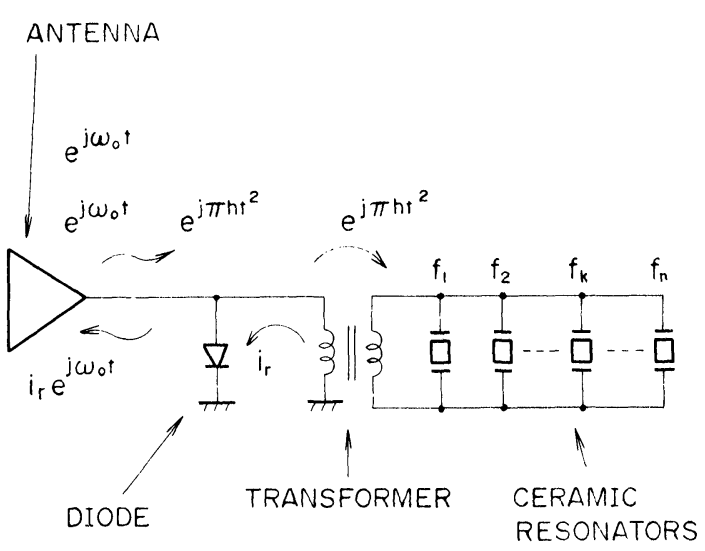

FIGURE 5 Transponder circuit model.

modulation on it according to the transponder's identification code. The transponder's circuit model is shown in Figure 5. It consists of an antenna, a diode, a transformer, and ceramic resonators. As shown in this figure, no oscillator or other means of generating a new signal is employed. One diode only is used to perform the demodulation and modulation function which will be described hereafter. The interrogating signal received by the transponder consists of the "carrier wave" component and the side-band component corresponding to the "sweep-frequency" signal. These can be represented by:

$$
\begin{array}{ll}
\text { Carrier wave component: } & \exp \left(j \omega_{0} t\right) \\
\text { Side-band component: } & \exp \left(j \omega_{0} t\right) \cdot \exp \left(j \pi h t^{2}\right)
\end{array}
$$

where, $\omega_{0}$ : carrier wave angular frequency. When these signal components are impressed on the diode, the diode will be excited by the carrier wave component $\exp \left(j \omega_{0} t\right)$ and as a result of its non-linearity, side-band components $\exp \left(j \omega_{0} t\right) \cdot \exp \left(j \pi h t^{2}\right)$ is demodulated, and the sweep-frequency signal $\exp \left(j \pi h t^{2}\right)$ is derived. This signal is applied to the ceramic resonators through the transformer, and make the ceramic resonators start "ringing" one by one successively at their own resonance frequencies. Referring to one of the ceramic resonators, the instant when the frequency of the sweep-frequency signal coincides with its resonance frequency, the resonator starts "ringing". The ringing lasts for a period determined by the $Q$-value of the resonator even after the sweep-frequency signal has passed the resonance frequency point.

Thus the ceramic resonators produce so called "ringing signals" which have different frequencies from each other corresponding to their own resonance frequencies, and as long as the "ringing" continues, the ringing signal currents will flow through the diode. The ringing current $i_{r}$ produced by the " $k$ "th ceramic 
resonator can be represented by:

$$
\begin{aligned}
i_{r}=\mathrm{I}_{k} \exp \left[\left(-\omega_{k} / 2 Q_{L}\right)\left(t-\tau_{k}\right) \cdot\right. \\
\exp \left[-j \pi h\left(t-\tau_{k}\right)^{2}\right] \cdot \exp \left(j \pi h t^{2}\right)
\end{aligned}
$$

where,

$$
\mathrm{I}_{k} \text { : constant }
$$

Since the diode is excited by the microwave carrier component $\exp \left(j \omega_{0} t\right)$ and is in a non-linear state, when ringing current $i_{r}$ flows through the diode, the microwave carrier will be amplitude-modulated by the ringing current and a modulated signal component $i_{r} \exp \left(j \omega_{0} t\right)$ will be generated. This signal component is transmitted through the antenna as the response signal.

Furthermore, the "ringing signal" of Eq. (2) is synchronously detected as the interrogator by using the sweep-frequency signal $\exp \left(j \pi h t^{2}\right)$ as the reference, and is converted to the "ringing beat" of the form shown in Eq. (1).

\subsection{Requirements for the Diode}

The diode in the transponder is used to modulate and demodulate the interrogating signal and the response signal by means of its non-linear characteristic.

Conversion efficiency of the diode depends to a high degree on the carrier wave power that excites it. As a system, a diode that requires a small current for its excitation is desirable and this is one of the factors in its selection. The dependency of the conversion efficiency on the excitation power is determined by the V-I characteristic, and the lower the barrier height the smaller the excitation current. In general the V-I characteristic of a diode can be represented by:

$$
\mathrm{I}=i_{s}\left(\mathrm{e}^{\alpha \mathrm{v}}-1\right)
$$

\section{$i_{s}:$ saturation current}

$\alpha$ : constant of the diode

If " $g$ " is the conversion conductance,

$$
g=\frac{d \mathrm{I}}{d \mathrm{~V}}=\alpha i_{s} \mathrm{e}^{\alpha \mathrm{V}}
$$

From the above the larger the value of $\alpha$ and V, the better the conversion efficiency rises, when the V-I curve becomes steeper, and the excitation voltage becomes higher. However, under actual conditions, Eq. (3) will not hold in the range of high diode current, and the non-linear characteristic disappears, when a certain voltage is exceeded, the conversion efficiency becomes constant and will not rise. Figure 6 shows typical V-I characteristics of various mixer diodes. Among these, those with the lowest barrier voltage values, or those that can be excited at low voltages are the "zero bias" Schottky diode. In this system, the zero bias Schottky

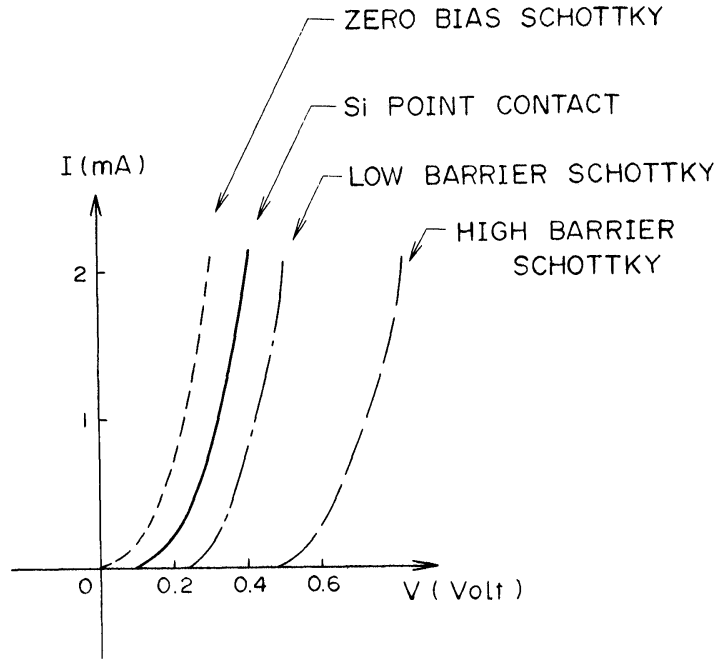

FIGURE 6 Typical V-I characteristics of various mixer diodes.

diode was used permitting proper functioning of the diode at a low power level of about $-15 \mathrm{dBm}$.

\subsection{Circuit Configuration}

The equivalent circuit of the transponder is shown in Figure 7. In this circuit, two elements that significantly affect the performance of the transponder are, the matching circuit; at the input of the diode, and the transformer; for coupling the ceramic resonator group.

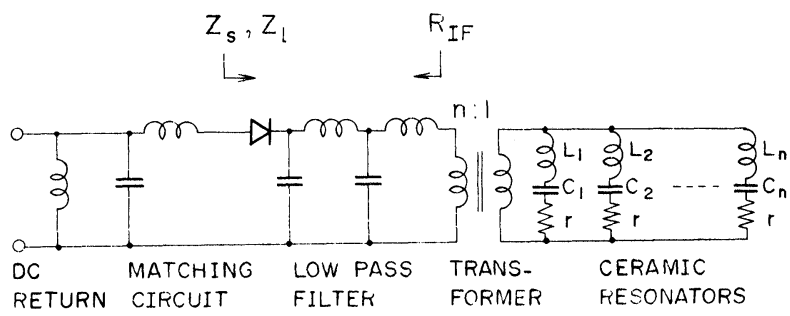

FIGURE 7 Transponder equivalent circuit.

4.2.1. The matching circuit The RF impedance of the diode is considered as $Z_{l}$ and $Z_{s} . Z_{l}$ is an impedance to the local oscillator signal or carrier wave, and $Z_{s}$ is an impedance to the modulated signal. Both of them are regarded as a function of the carrier wave's power level. In the usual mixer circuit, the local signal level is large enough to drive a diode and constant, so that the matching circuit is designed to match $Z_{s}$. However, since this system must be designed to function with a low level and varying carrier wave power, the matching circuit should be designed to match $Z_{l} . Z_{l}$ is a function of the carrier wave's power level, and for the "zero 
bias" Schottky diode this level is around $-15 \mathrm{dBm}$. Normally, the values of $Z_{s}$ and $Z_{l}$ are approximately equal at the same frequency so that when matching is obtained for $Z_{l}$, matching for the signal is sufficient for all practical purposes.

4.2.2. Transformer Impedance matching between the diode and the resonator group is desirable for maximum transfer of the demodulated sweep frequency signal energy, to obtain maximum resonating current. This resonating current has an amplitude that decays exponentially with $\exp \left(-\omega_{\mathrm{k}} / 2 Q_{L}\right) \mathrm{t}$, so that a value of $Q_{L}$ is sufficiently high to produce ample ringing is desirable. Proper matching and the value of $Q_{L}$ is determined by the design of the transformer. The " $k$ "th resonating element is represented in the resonance circuit model shown in Figure 8 here, current build up starts at time $t=\tau_{k}$ and the resonance current $\mathrm{I}_{r}$ can then be represented as,

$$
\mathrm{I}_{r}=\frac{E_{s} n}{R_{\mathrm{IF}}+r n^{2}}
$$

where,

$E_{s}:$ voltage of the sweep frequency signal

$R_{\mathrm{IF}}$ : IF impedance of the diode resonance resistance of the resonator

$n$ : transformer turn ratio

Next, at time $t>\tau_{k}$ after the sweep-frequency signal has shifted, resonance current still flows in the circuit. If the $Q$ value of the circuit is $Q_{L}$ at this time, then,

$$
Q_{L}=\frac{Q_{0} r n^{2}}{R_{\mathrm{IF}}+r n^{2}}
$$

where,

$$
Q_{0} \text { : unloaded } Q \text { of the resonator }
$$

Relationship between the transformer ratio, the resonance current $I_{r}$ and $Q_{L}$ is shown in Figure 9 . Observing $I_{r}, I_{r}$ takes on maximum value when

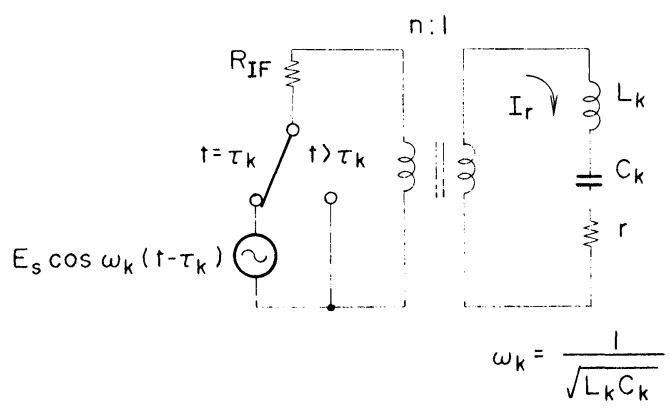

FIGURE 8 Resonance circuit model.

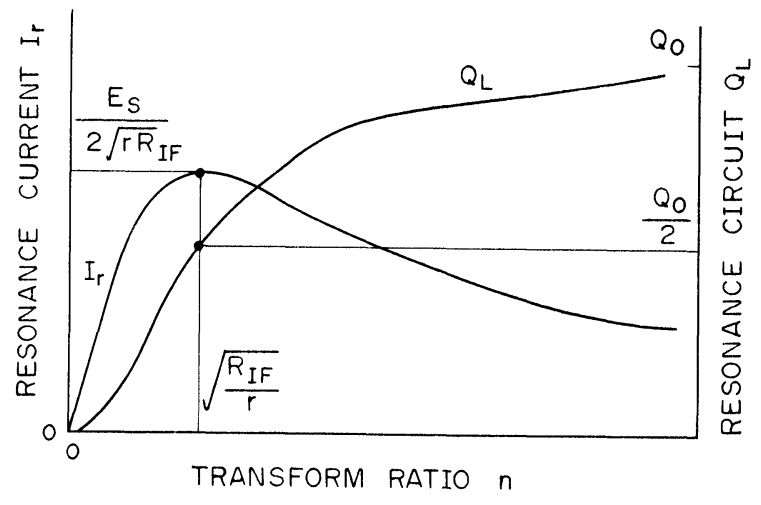

FIGURE 9 Transform ratio $n$ vs. resonance current $I_{r}$ and resonance circuit $Q_{L}$.

$n=\sqrt{R_{\mathrm{IF}} / r}$. Observing $Q_{L}$, value of $Q_{L}$ increases with that of $n$ and approaches $Q_{0}$. Where $I_{r}$ is maximum, $Q_{L}=Q_{0} / 2$. Therefore, if the value of $Q$ is sufficiently large, then the optimum value for " $n$ " will be $\sqrt{R_{\mathrm{IF}} / r}$. At this time, $Z_{\mathrm{IF}}$ will be a function of the carrier wave's power level just as $Z_{s}$ and $Z_{l}$, and $Z_{\mathrm{IF}}$ will increase when the power level decreases. Thus it becomes necessary to determine transformer ratio " $n$ " in accordance with the value of $Z_{\mathrm{IF}}$ at low power level, the same as for the "matching circuit".

\subsection{Conversion Loss of the Transponder}

The conversion loss is defined as the ratio between the interrogating signal and the response signal at the input of the transponder circuit. Putting the power level of the interrogating signal as $P\left(f_{0} \pm f_{s}\right)$, and the power level of the response signal or, the "ringing modulated signal" as $P\left(f_{0} \pm f_{r}\right)$. Then, the conversion loss $L_{c}$ can be given by:

$$
L_{c}=10 \log \frac{P\left(f_{0} \pm f_{r}\right)}{P\left(f_{0} \pm f_{s}\right)}(\mathrm{dB})
$$

Actual transponder conversion loss data is presented in Figure 10. The data indicates that the transponder is capable of operating with a carrier wave power level of as low as $-15 \mathrm{dBm}$.

\subsection{Encoding by Ceramic Resonators}

This system uses a sweep-frequency signal in the mediam wave-band $(855$ to $2450 \mathrm{kHz})$, and a ceramic resonator with a $Q$-value of about 1000 . Individual identification coding of each transponder is achieved by a combination of the frequencies of the ceramic resonators. Because individual identification codes are, 


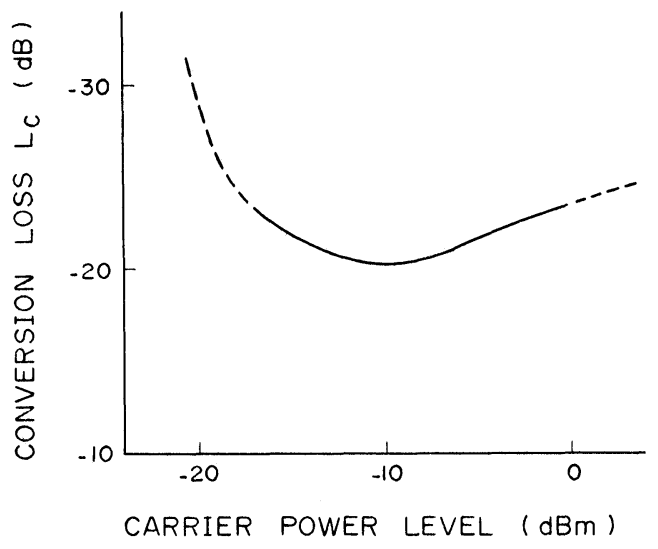

FIGURE 10 Transponder conversion loss.

normally, designated in decimal numbers, each decimal resonators, and in order to hold the number of ceramic resonators to a minimum, ${ }_{5} \mathrm{C}_{2}$ encoding is employed. Table $\mathrm{I}$ is the ${ }_{5} \mathrm{C}_{2}$ encoding system used in this system. Each digit is given 5 different resonance frequencies and by selecting 2 resonance frequencies out of the five frequencies, a decimal number is expressed. Therefore, in order to represent an $N$ digit decimal number, $5 N$ kinds of resonance frequencies will be necessary. Table II is an example of the "frequency allocation" for the transponder of seven decimal digits.

TABLE I

${ }_{5} \mathrm{C}_{2}$ Encoding.

\begin{tabular}{|c|c|c|c|c|c|c|c|}
\hline (a) Weight & & (b) Frequ & ency & om & natic & & \\
\hline & & & & esp & $\begin{array}{l}\text { ding } \\
\text { on }\end{array}$ & requ & \\
\hline $\begin{array}{l}\text { Frequency } \\
\text { per digit }\end{array}$ & Weight & number & f1 & f2 & $\mathrm{f} 3$ & $\mathrm{f} 4$ & f5 \\
\hline f1 & 0 & 1 & 0 & 0 & & & \\
\hline $\mathrm{f} 2$ & 1 & 2 & 0 & & 0 & & \\
\hline f3 & 2 & 3 & & 0 & 0 & & \\
\hline $\mathrm{f} 4$ & 3 & 4 & 0 & & & 0 & \\
\hline f5 & 4 & 5 & & 0 & & 0 & \\
\hline & & 6 & & & 0 & 0 & \\
\hline & & 7 & 0 & & & & 0 \\
\hline & & 8 & & 0 & & & 0 \\
\hline & & 9 & & & 0 & & 0 \\
\hline & & 0 & & & & 0 & 0 \\
\hline
\end{tabular}

TABLE II

Transponder frequency allocation.

\begin{tabular}{llllll}
\hline \multicolumn{7}{c}{ Frequency } \\
\cline { 2 - 6 } Digit & f1 & f2 & f3 & f4 & f5 \\
\hline \#1 Check digit & 1115 & 1130 & 1145 & 1160 & 1175 \\
$\# 2\left(10^{0}\right)$ & 1191 & 1207 & 1223 & 1239 & 1255 \\
$\# 3\left(10^{1}\right)$ & 1272 & 1289 & 1306 & 1323 & 1340 \\
$\# 4\left(10^{2}\right)$ & 1358 & 1376 & 1394 & 1412 & 1430 \\
$\# 5\left(10^{3}\right)$ & 1449 & 1468 & 1487 & 1506 & 1525 \\
$\# 6\left(10^{4}\right)$ & 1546 & 1567 & 1588 & 1609 & 1630 \\
$\# 7\left(10^{5}\right)$ & 1652 & 1674 & 1696 & 1718 & 1740 \\
\hline
\end{tabular}

\subsection{Antenna}

It is most desirable for the antenna of this system to be flat, compact, and light weight, because the transponder's construction will be largely affected by the design of its antenna. The "printed slot antenna" is ideally suited for this system. Its construction consists essentially of a sheet of dielectric substrate $(0.35 \mathrm{~mm}$ thick PTFE-glass fibre), on one side of which a slot $(1 / 2$ carrier wavelength long), is provided. The other side of the substrate sheet has a "feed line" formed by a microstrip line. The antenna developed for this system's transponder is a printed slot array antenna, having two slots, and with a reflector plate spaced $7 \mathrm{~mm}$ from the substrate. Main specifications are:

$\begin{array}{ll}\text { Centre frequency: } & 2.45 \mathrm{GHz} \\ \text { Gain: } & 7.5 \mathrm{~dB} \\ \text { Beam width: } & 50^{\circ} \times 75^{\circ} \\ \text { Dimensions: } & 64 \times 96 \times 7 \mathrm{~mm}\end{array}$

\subsection{Construction of the Transponder}

All of the circuitry required for the transponder is provided in the form of microstrip lines utilizing MIC (Microwave Integrated Circuit) technology mounted on the antenna substrate. The antenna and the transponder circuitry are patterned as an integral unit on the single substrate. No adjustments are required so that the unit is easy to produce in large quantities. Furthermore, no connections other than for the diode, transformer, and ceramic resonators mounted on the substrate require soldering so that a high degree of reliability can be expected. The circuit pattern developed for the transponder of this AVI system is shown in Figure 11, and the assembly is shown in Figure 12 . The identifying information capacity for this unit, consists of seven digits of decimal number. An external view of the transponder is shown in Figure 13. The antenna substrate, and reflector plate are 


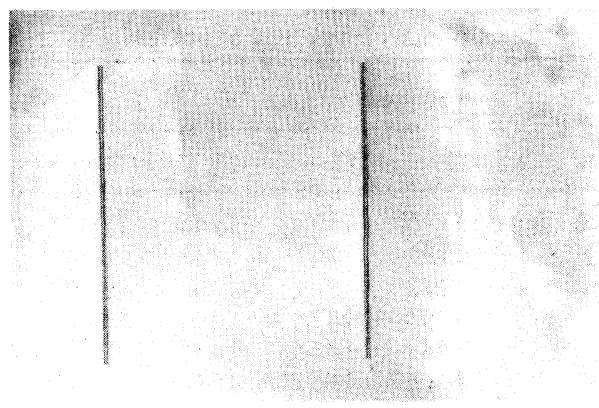

Fore (slotted antenna)

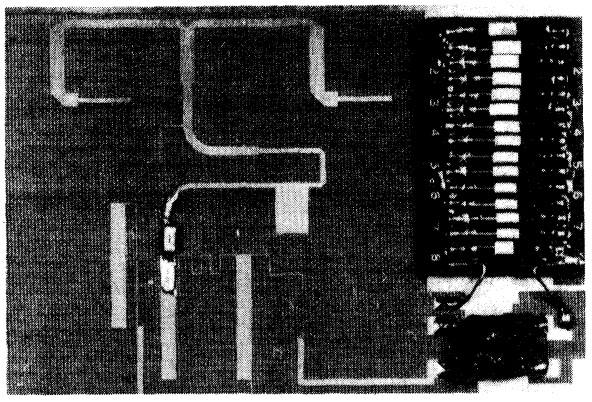

Back (feeder and circuit pattern)

FIGURE 11 Transponder circuit pattern.

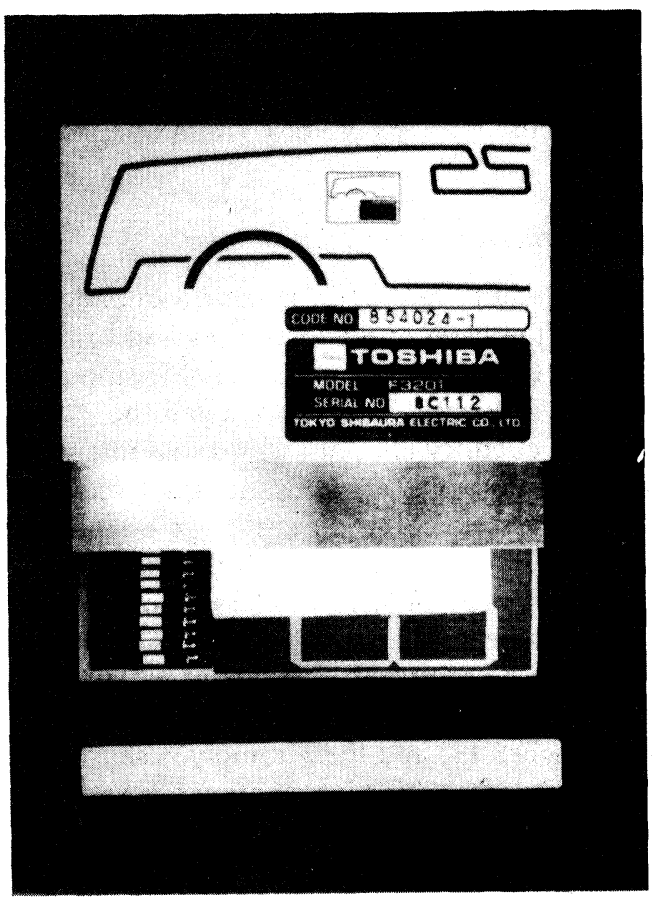

FIGURE 12 Transponder assembly.

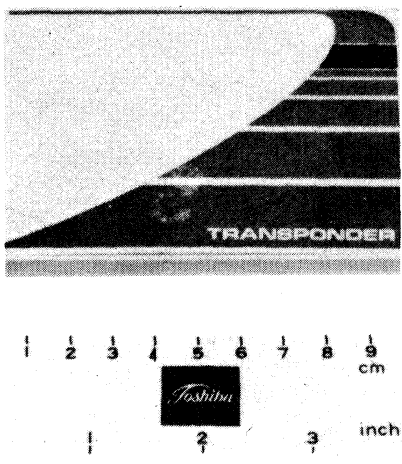

FIGURE 13 AVI transponder.

contained in a plastic case. Overall external dimensions are $7 \times 10 \times 1 \mathrm{~cm}$ with a weight of 150 grams, the unit is compact, light weight and can be mounted easily on the vehicle, and is easy to carry.

\section{CONCLUSION}

The AVI transponder described in this paper is characterized by no-battery operation and simple construction which based on its unique operation principle, and by small light weight, and compactness which are realized by use of MIC (Microwave Integrated Circuit) and HIC (Hybrid Integrated Circuit) technology. This has enabled the transponder to have a wide range of applications in the automatic vehicle identification systems.

\section{REFERENCES}

1. H. Murakami et al., "Automatic Car Identification System Using Electromagnetic Induction Coupling," Toshiba Review, (March 1972).

2. S. Kurotori et al., "New Tokaido Line Train Number Identification System," Toshiba Review, (Sep.-Oct. 1974).

3. "Identification of Wagon Numbers," ORE Report, Question A 97, (Oct. 1967).

4. "ACI meets its crucial tests," Railway Signaling and Communications, pp. 19-20, (Dec. 1966).

5. "Competing ACI systems evenly matched in UIC reading tests," International Railway Journal, pp. 56-59, (Nov. 1971).

6. W. Linhardt, "Microwave Car Identification System Siemens SICARID," International Railway Journal, pp. 57-58, (Nov. 1971).

7. R. J. Klensch et al., "A Microwave Automatic Vehicle Identification System," RCA Review, 34, pp. 566-579, (Dec. 1973).

8. R. A. Hauslen, "The Promise of Automatic Vehicle Identification," IEEE Trans. Veh. Technol., VT-26, pp. 30-38, (Feb. 1977).

9. J. Sakuragi et al., "Microwave Automatic Vehicle Identification (AVI) System" 29th IEEE Vehicular Technology Conference, pp. 72-78, (March 1979). 

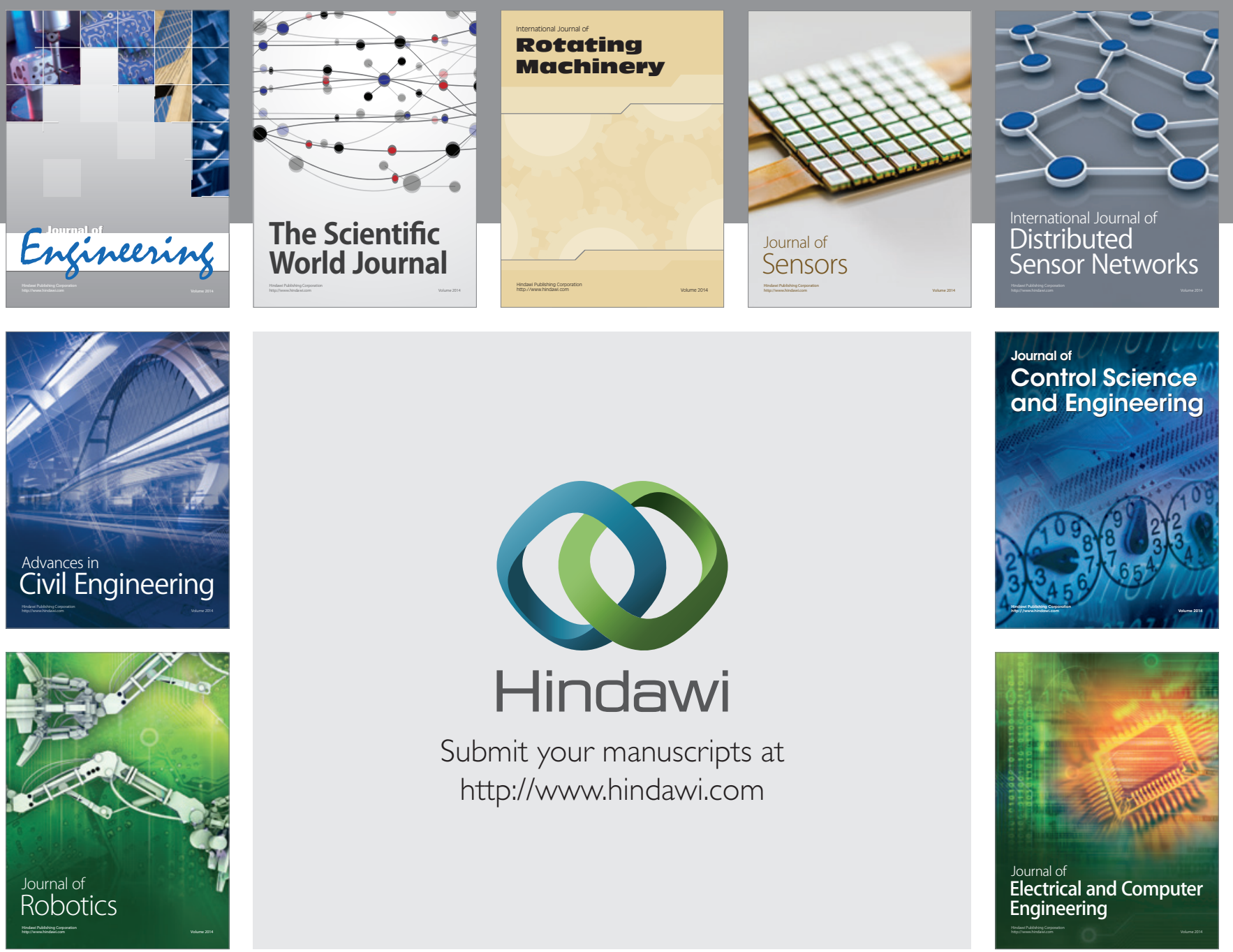

Submit your manuscripts at

http://www.hindawi.com
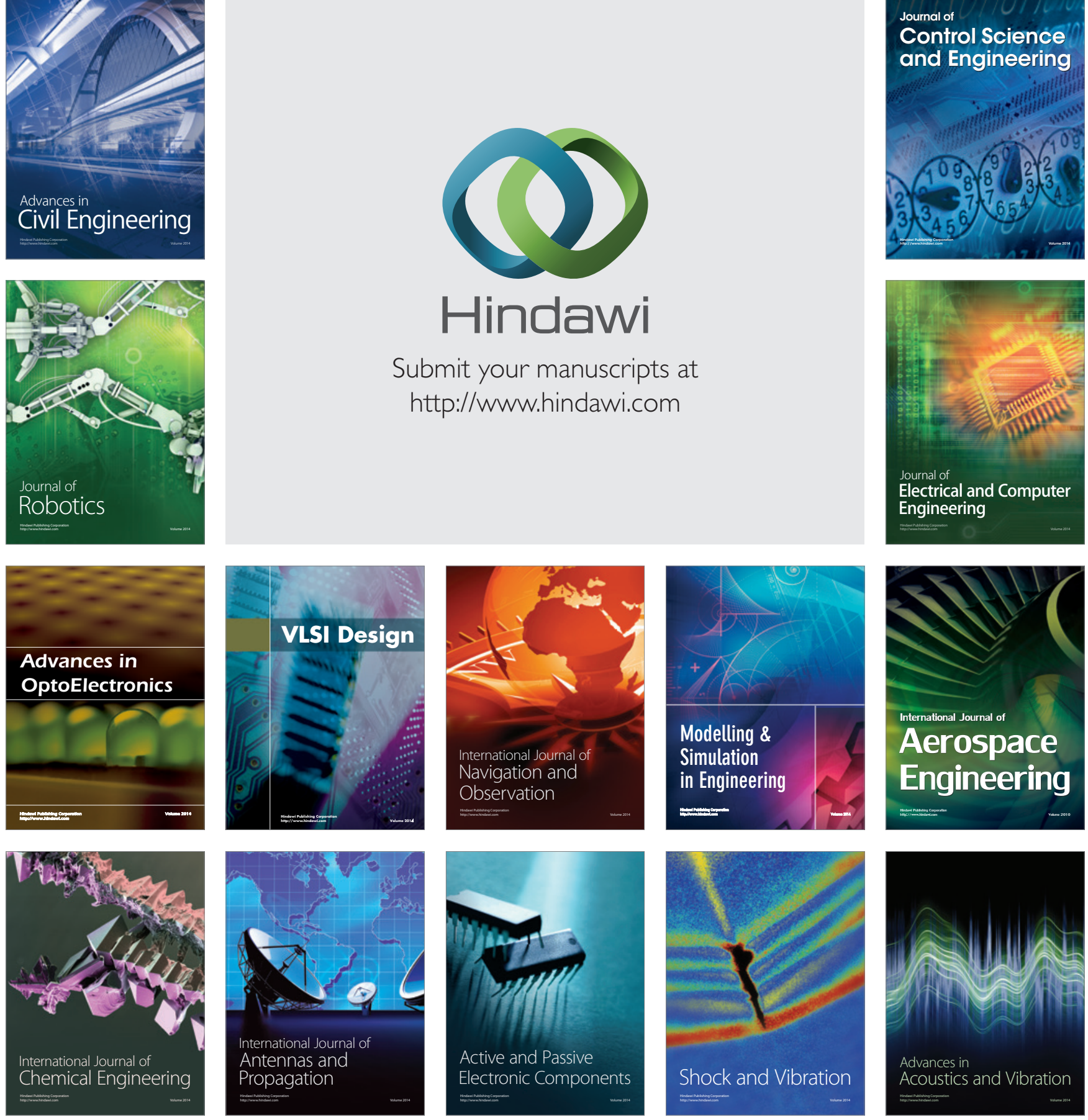